\title{
Wie Nathan der Weise nach Israel kam
}

\author{
Szenische Interpretation eines klassischen deutschen Dramas \\ in einer multikulturellen und multilingualen Lerngruppe. Ein \\ Bericht 1/4ber die Projektarbeit.
}

\section{Brigitte Hahn-Michaeli}

\begin{abstract}
Zusammenfassung
Angehende Naturwissenschaftler und Ingenieure im DaF-Unterricht in Israel $\mathrm{f}^{1} / 4 \mathrm{r}$ deutsche klassische Literatur zu motivieren scheint ein kaum zu erf $1 / 4$ llender, hoher Anspruch. Unter den gegebenen Rahmenbedingungen in der Lerngruppe von drei unterschiedlichen Kulturen und Muttersprachen zur Interaktion und Kommunikation zu bewegen, stellt eine zusatzliche Herausforderung dar und wirft die Frage auf, welche Formen der methodischen Vermittlung, die das Potenzial einer multikulturellen Lerngruppe ber $1 / 4$ cksichtigen, zur literarischen Textarbeit motivieren und effektiv eingesetzt werden $\mathrm{k} \boldsymbol{\top}$ nnen. Herk $\boldsymbol{\top}$ mmliche Arbeitsweisen, bei denen die Literaturvermittlung vorwiegend durch kognitive Analyse vollzogen wird, erscheinen unter diesen Gegebenheiten unzureichend. Hingegen bietet die aus der Theaterarbeit entlehnte dramapodagogische Ver-

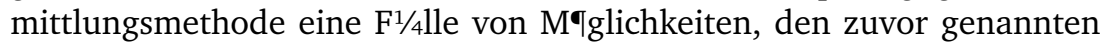
Anforderungen gerecht zu werden und dabei den interkulturellen Dialog durch kreative und aktive Auseinandersetzung mit der Literatur zu f -rdern. In diesem Beitrag soll die Vorbereitung und Durchf $1 / 4$ hrung eines Semesterprojekts mit Lessings Nathan der Weise unter Einsatz unterschiedlicher Dramatechniken beschrieben werden. Bei dieser Form des ganzheitlichen, interaktiven Lernens wird nicht nur die Begegnung mit dem literarischen Text erm $\mid$ glicht und zur Reflexion angeregt, es findet gleichzeitig eine intensive interkulturelle Auseinandersetzung in der Lerngruppe statt.
\end{abstract}

\section{Wie Nathan der Weise nach Israel kam und die Studenten zu Nathan der Weise}

Klassische Literatur des 18. Jahrhunderts mit Studenten im Kurs Deutsch als Fremdsprache an einer Technischen Universitot in Israel? Ein Gedanke, der mir zun, chst unrealistisch und nicht durchf $1 / 4$ hrbar schien; unter den gegebenen Lern- und Lehrvoraussetzungen $\mathrm{w}^{1 / 4} / 4$ de eine unterrichtliche Umsetzung in 
jedem Falle eine enorme Herausforderung bedeuten in dem Land, in dem Nathan der Weise beheimatet war und in dem religi $\boldsymbol{\top}_{\text {se }}$ und kulturelle Konflikte das alltogliche Leben heute wie schon vor Jahrhunderten beeinflussen. Fast $1 / 4$ berall in Israel leben Juden, Araber (Muslime und Christen), sowie Drusen mit wenigen Ausnahmen roumlich streng voneinander getrennt und die Sch1/4ler werden an getrennten Schulen bis zur Hochschulreife unterrichtet. Eine der wenigen M厅glichkeiten zur Begegnung und zu kooperativem Lernen kann

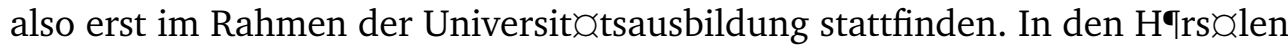
verschwindet die bestehende gesellschaftliche Problematik unter dem Deckmantel der Masse und der Anonymitort. In einem kommunikativ orientierten Fremdsprachenunterricht bildet jedoch diese distanzierte, oftmals unnahbare Haltung von Muslimen, Juden und Christen, Arabisch-, Hebrøisch- und Russischmuttersprachlern eine $\mathrm{H}^{1} / 4$ rde, die zun 0 chst $1 / 4$ berwunden werden muss, um eine Annoherung und damit eine Basis zur gemeinsamen Kommunikation zu schaffen.

Die Lernergruppe, $\mathrm{f}^{1} / \mathrm{r}$ die ich das Projekt plante, bestand aus Studenten, die im Rahmen eines studienbegleitenden Wahlpflichtkurses im dritten Semester DaF lernten. Somit kam nicht in Frage, den Text in der Originalfassung zu lesen. Es musste also von einer vereinfachten Textform ausgegangen werden, die den Studenten eine Auseinandersetzung mit dem Inhalt des Dramas erm $\boldsymbol{\top}$ glichte und sie beforhigte, den Inhalt in der Fremdsprache und in eigenstơndiger Interpretation und szenischer Gestaltung darzustellen. Inwieweit die Studenten $1 / 4$ ber die sprachliche Interpretation hinaus Bereitschaft und Interesse zeigen $\mathrm{w}^{1} / 4$ rden, auch an der Thematik des Dramas zu arbeiten und sich damit auch auf eine interkulturelle Auseinandersetzung mit dem Inhalt des St $1 / 4$ ckes einzulassen, war zu Beginn der Projektarbeit nicht einsch atzbar. Meine Erfahrungen, die ich diesbez $1 / 4$ glich im DaF-Unterricht an der Technischen Universitót erlangen konnte, zeigten mir, dass Studenten der Natur-/ Ingenieurwissenschaften leider nur geringes Interesse an literarischer Textinterpretation hatten. Es stellte sich nun die spannende Frage, inwieweit die Bearbeitung einer Thematik, die einen sehr direkten Bezug zu der aktuellen Situation der Studenten hier im Land haben $\mathrm{k} \boldsymbol{\top} n n t e$, in inhaltlich-interkultureller Hinsicht zu neuen Einsichten und Erkenntnisgewinn $\mathrm{f}^{1 / 4} / \mathrm{hren} \mathrm{w}^{1 / 4} \mathrm{rde}$.

Mit diesem Projektbericht $\mathrm{m}$ Tchte ich beschreiben, wie in einem multikulturellen und multilingualen Lernumfeld durch den Einsatz von dramapodagogischen Mitteln ein aktiver Umgang mit literarischen Texten und somit die Interaktion in der Klasse stark gef rdert werden kann. Zun $\measuredangle$ chst aber soll eine kurze Bestandsaufnahme die Lehr- und Lernvoraussetzungen an einer israelischen Universit $₫ \mathrm{t}$ erløutern. Danach erfolgt eine nơhere Betrachtung des Textes mit kurzer Inhaltsangabe, der sich berlegungen $\mathrm{f}^{1 / 4} \mathrm{r}$ den Einsatz im Unterricht anschlieen. Die Beschreibung der Projektarbeit und die dabei gemeinsam gewonnenen Erfahrungen werden abschlieend ausgewertet und reflektiert.

Somit versteht sich dieser Aufsatz als Denkansto, als Inspiration $\mathrm{f}^{1} / 4 \mathrm{r}$ Lehrende, die unter ohnlichen Lehr- und Lernvoraussetzungen arbeiten, erhebt aberkeinen Anspruch auf allgemeine G1/4ltigkeit. Indem meine Studenten und ich unseren 
Klassenraum im 'Technion' zur B1/4hne des Lebens umdefinierten und der M厅glichkeit zur zwischenmenschlichen Begegnung in der Fremdsprache Raum schafften, $\boldsymbol{\top}_{\mathrm{ffn}}$ ten wir uns einen neuen Weg zur Kommunikation, der in seiner Unvorhersehbarkeit spannend war, der den Studenten die Kenntnisse einer Fremdsprache verschaffte, ohne dass sie einem trockenen Vermittlungsprozess ausgesetzt wurden und der uns vor allen Dingen (fast) immer sehr viel Spa machte. Grund genug, auf diesem Weg weiter $\mathrm{zu}$ forschen und $\mathrm{zu}$ experimentieren. Jedoch darf die berzeugung, dass wir mit Dramapodagogik im Fremdsprachenunterricht auf dem einzig richtigen Weg sind, die Lernenden nicht 1/4berrollen. Bei dieser Art von Arbeit, bei der es vorwiegend um die Begegnung mit dem Selbst, dem eigenen Ich und dem Partner, dem Fremden und Unbekannten geht, $\mathrm{m}^{1 / 4} \mathrm{ssen}$ wir stondig wach bleiben und nicht nur den aueren, sondern auch den inneren Dialog lebendig und im aktiven Austausch halten. Die Gruppendynamik bei der Theaterarbeit zeichnet sich dadurch aus, dass man auf Zusammenarbeit angewiesen ist. Und wenn mir meine Studenten signalisieren, dass sie mit meiner ach, so gut geplanten und didaktisierten Idee im Moment nichts anfangen $\mathrm{k} \boldsymbol{\top} \mathrm{nnen}$, so muss ich als Lehrende und Initiatorin ebenso kreativ und spontan umschalten und umdisponieren $\mathrm{k} \boldsymbol{\top} n n e n$, wie ich es von meinen Lernenden im Unterricht erwarte. Theaterarbeit ist verf $1 / 4$ hrerisch in ihrer Attraktivitoxt, darf sich aber im Fremdsprachenunterricht genauso wenig verselbststơndigen, wie jedes andere Unterrichtsverfahren. Jeder kann nur $\mathrm{f}^{1} / 4 \mathrm{r}$ sich und seine Lerngruppe die passenden Elemente herausfinden und 1/4bernehmen und durch das eigene Erproben die individuellen M-glichkeiten und Grenzen erfahren.

\section{Die Lern- und Lehrvoraussetzungen}

Als ich vor fast 20 Jahren meine Lehrtotigkeit als DaF-Dozentin am Technion aufnahm, fand ich in meinen Klassen ein Mosaik deutlich voneinander abgegrenzten Kleingruppen vor. Juden, Muslime, Drusen und Christen mit den Muttersprachen Hebrøisch, Arabisch und Russisch zeigten eine starke Tendenz, ausschlielich innerhalb der kulturellen oder linguistischen Kleingruppe, der sie angeh $\boldsymbol{\top}$ rten, zu arbeiten. Diese bestehende mangelnde Bereitschaft zur Kommunikation und Zusammenarbeit lie mich nach alternativen Lehrmethoden suchen, deren Einsatz im DaF-Unterricht den interkulturellen Dialog f rdern $\mathrm{k}$-nnten. Auf einem Fortbildungsseminar in Deutschland befasste ich mich zum ersten Mal mit dramapodagogischer Vermittlungsmethodik im Fremdsprachenunterricht und erkannte den auerordentlichen Wert dieser Unterrichtsform und die Bereicherung, die damit in den Fremdsprachenunterricht eingebracht werden kann. $\mathrm{F}^{1 / 4} \mathrm{r}$ meine Studenten war diese Form von aktiver und ganzheitlicher Fremdsprachenvermittlung zunøchst gonzlich ungewohnt und stellte offensichtlich eine befremdliche Lernsituation dar, so dass eine schrittweise Annoherung an die neue Vermittlungsmethode $\mathrm{n}$ Itig war. Ich begann in meinen Anfơngerklassen zunehmend Aktivit@oten zu integrieren, die aus der Theaterarbeit entlehnte Elemente beinhalteten und konnte feststellen, wie die 
Kommunikationsfohigkeit der Studenten dadurch erheblich verbessert wurde. Spielen und Experimentieren brachte eine zuvor unbekannte Lebendigkeit ins Unterrichtsgeschehen, lud zum kreativen Ausdruck ein und nahm vor allen Dingen die oftmals lohmende Angst etwas 'Falsches' zu sagen. Die Form der interaktiven Arbeit trug dazu bei, Hemmungen zu beseitigen und die aktive Zusammenarbeit von Studenten mit linguistisch und kulturell unterschiedlichen Hintergr ${ }^{1 / 4} / 4$ den zu f $\boldsymbol{f}$ rdern. Dem unbekannten Gegen $1 / 4$ ber in der Klasse, sei es in Form der anderen Muttersprache, der anderen Religions- oder Kulturzugeh Trigkeit, vor dem man bisher Scheu gezeigt hatte, konnte man durch die unterschiedlichen bungen und Aktivitoten begegnen und sich ganz im Sinne des folgenden Zitats dem Anderen ohne Angst annơhern:

Fremdheit ist also keine objektive Eigenschaft, sondern eine Frage der Perspektive [...]. In diesem Sinne kann interkulturelle Theaterarbeit die Ziele der interkulturellen Podagogik (Akzeptieren, Respektieren und Aushalten des Anderen) aufnehmen und handlungspraktisch umsetzen, ohne ihre theaterspezifischen Momente des spielerischen Experiments, der bertreibung und Verfremdung oder auch der Irritation aufgeben $\mathrm{zu} \mathrm{m}^{1 / 4} / 4 \mathrm{ssen}$. Vielmehr stellen sich im Theaterprozess auf unterhaltsame Weise sowohl kognitive und sinnlich-emotionale als auch fremde und befremdende Erfahrungen, eben Differenzerfahrungen ein. Differenzerfahrung, die nicht von Angst begleitet ist, ist ein positives Kulturerlebnis. (Sting 2003: 1f)

Von auerordentlich groer Bedeutung bei dieser Arbeitsform der szenischen Interpretation sind die roumlichen Gegebenheiten. Tisch- und Stuhlreihen, wie man sie normalerweise in jedem Klassenraum vorfindet, bilden kommunikationshemmende Barrieren. Es muss also zun đochst Raum geschaffen werden, der 1/4berhaupt erst den oueren Rahmen zur Begegnung und Kommunikation zuløsst. Schwerdtfeger (2002: 7) beschreibt die wichtige Bedeutung von Raum und ihren Einfluss auf die menschliche Begegnung:

Das soziale, das sprachliche und nicht-sprachliche Verhalten der Menschen wird also stark bestimmt von dem Arrangement der Einrichtung, die sie in einem Raum vorfinden oder selbst schaffen. .... Wir finden in einem Raum eine Anordnung von MTbeln vor und diese Anordnung scheint die soziale Organisation, d.h. eine soziale Anordnung von Menschen, die sich in diesem Raum befinden werden, festzulegen und damit immer auch ihre MIglichkeit, sprachlich und nicht-sprachlich Kontakt aufzunehmen.

Schon in den Anfơngerkursen wurden die Studenten an die Arbeit im 'leeren Raum' gew $T h n t$, der einen personen- und gruppenorientierten Unterricht erm Iglichte, eine Perspektive $\mathrm{f}^{1 / 4} \mathrm{r}$ authentische Kommunikationssituationen -ffnete und zur Begegnung herausforderte. Nur unter diesen Rahmenbedingungen kann Fremdsprache annohernd authentisch wie im wirklichen Leben verbunden mit Mimik, Gestik und Kगrperbewegung im Raum zum Ausdruck kommen, und der Lernstoff wird nicht als isoliertes Element vermittelt. 


\section{Die verơnderte Lehrerrolle}

Der dramap@dagogisch orientierte Fremdsprachenunterricht erfordert eine Neudefinierung der Lernerrolle, die unterschiedliche Anforderungen an die Lehrperson stellt. Im traditionellen Fremdsprachenunterricht ist die Lehrperson vorwiegend Wissensvermittlerin und alleine verantwortlich $\mathrm{f} 1 / 4 \mathrm{r}$ die Unterrichtsgestaltung. Die Dramapodagogik spricht den Lerner jedoch in seiner ganzen Person an und bezieht ihn somit viel storker in den Lernprozess mit ein. Die Dynamik, die sich bei dieser Art von Arbeit im Klassenraum entwickelt und den eigentlichen Lernprozess ausmacht, rangiert vor der fachlichen Vermittlung von Lerninhalten. Der Lehrperson stellt sich in diesem neuen Rahmen die Herausforderung, gruppendynamische Aktivitoten zu initiieren und so kommunikative Lernprozesse zu f $\boldsymbol{\top}$ rdern. Dies setzt jedoch voraus, dass seitens der Lehrperson die Bereitschaft vorhanden ist, Autoritot aufzugeben und lernerzentrierte Arbeitsformen einzubringen. Der Anspruch, dass die Lerner fehlerfrei und grammatisch korrekt sprechen kann bei dieser Form der vorwiegend kreativen Arbeit nicht aufrecht erhalten werden, es entsteht allerdings ein Freiraum f1/4r spontane Kommunikation und freien Ausdruck. Aktivitoten $\mathrm{k} \boldsymbol{\top}$ nnen durch die oftmals nicht vorhersehbare Gruppendynamik im lernerzentrierten Unterricht in ungeplante Richtungen steuern. Somit muss die Lehrperson flexibel bleiben und offen sein $\mathrm{f}^{1} / 4 \mathrm{r}$ neue Impulse, die sich im Arbeitsprozess mit den Lernenden

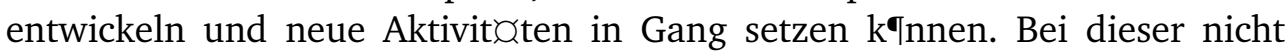
mehr ausschlielich seitens der Lehrperson gesteuerten Arbeit werden Barrieren zwischen Lehrperson und Lernenden abgebaut. Hemmungen mit dem Lehrer zu kommunizieren $\mathrm{k} \boldsymbol{\top}$ nnen nur dann entfallen, wenn der Lehrer nicht lơnger roumlich getrennt auf dem Podium vor der Klasse steht, sondern sich frei zwischen den Lerngruppen hin und her bewegt. Auch lernschwochere Studenten ergreifen dann das Wort und bringen sich in das Gruppengeschehen ein. Baur (1990: 30, zitiert in Schewe 1993: 212f) betont die Wichtigkeit von Gruppenarbeit und Bewegung im Raum:

Es $\mathrm{m}^{1 / 4} / 4$ ssen deshalb Sozialformen des Lernens praktiziert werden, die [...] Angstemotionen reduzieren. Das gelingt unserer Erfahrung nach am besten, wenn die Interaktionsformen mit viel Bewegung, mit stơndig variierenden Gruppenkonstellationen und mit einem betont spielerischen Charakter verbunden werden. Dadurch nehmen die Teilnehmer einer Lerngruppe alle sofort pers nlichen Kontakt miteinander auf, und das $\mathrm{Gef}^{1 / 4} / 4 \mathrm{hl}$, von der Gruppe unterst $1 / 4$ tzt zu werden, tritt an die Stelle des $\mathrm{Gef}^{1} / 4 \mathrm{hls}$, beobachtet zu werden und sich bewohren oder behaupten zu $\mathrm{m}^{1 / 4} / 4 \operatorname{ssen}[\ldots]$

\section{Vor $1 / 4$ berlegungen zu dem Text Nathan der Weise}

Diemangelnde Motivation beijungen Leuten $\mathrm{f}^{1 / 4} \mathrm{r}$ literarische Texte unterscheidet sich in Israel wohl kaum vom Rest der Welt. Es besteht geringes Interesse an Literatur, niemand hat Zeit zum Lesen und die Medien Film und Internet 
dominieren mit ihrer 1/4berwoltigenden Bilder- und Informationsflut. Dennoch habe ich immer wieder mit kurzen, kleineren Texten, Prosa wie Lyrik gearbeitet, mich aber nie an einen longeren Text, gar ein ganzes Drama gewagt. Was sprach aber gegen den Versuch, mit einer vereinfachten Textfassung von Nathan der Weise zu arbeiten? Theoretisch konnte ich mir keinen geeigneteren Ort $\mathrm{f}^{1} / 4 \mathrm{r}$ die Bearbeitung dieses klassischen Dramas als den meiner multikulturellen und multilingualen Lerngruppe vorstellen. Das Thema, mit dem Lessing uns ins mittelalterliche Palostina $\mathrm{f}^{1} / 4 \mathrm{hrt}$, hat heutzutage eine ebenso starke Relevanz wie damals und bietet sich deshalb auch $\mathrm{f} 1 / 4 \mathrm{r}$ die Bearbeitung im DaF-Unterricht an.

Wenn hat, und wo die fromme Raserei

Den besseren Gott zu haben, diesen besseren

Der ganzen Welt als Besten aufzudringen

In ihrer schworzesten Gestalt sich mehr gezeigt

Als hier, als itzt? (2. Aufzug, 5. Auftritt)

So die Worte Lessings aus dem Jahr 1778/79. Fast 230 Jahre spoter bleibt die philosophische Fragestellung die gleiche, heutzutage lediglich in anderer Wortwahl. Die Thematik der verworrenen Familiengeschichte des Dramas, in dem Vertreter des Christentums, des Islam und des Judentums verwickelt werden, wiederholt sich heute in moderner Version in jeder Telenovela, die in ihrer Struktur den Lernenden zu Gen¹/4ge bekannt sind. Hatte ich anfounglich noch wegen der verworrenen und sehr konstruiert wirkenden Familiengeschichte in Lessings Text Bedenken, so 1/4berzeugte mich die stark thematische Relevanz der philosophischen Frage des Dramas. Das ber $1 / 4$ hmte Kernst ${ }^{1 / 4} 4$ ck des Dramas, die Ringparabel, stellt uns vor die Frage, welche der Weltreligionen die wahre und die richtige sei; eine Streitfrage, die endlose Konfliktsituationen in Israel ausl $\boldsymbol{\top}_{\text {st }}$ und im alltoglichen Leben in diversen Ausdrucksformen stondig prosent ist. Die R $1 / 4$ ckmeldung der Studenten in den Frageb Igen zur Projektarbeit am Semesterende bestotigte meine Annahme, dass nicht nur die verworrene Familiengeschichte mit viel Fantasie aufgenommen und in eigene Interpretationen umgesetzt wurde, sondern dass auch der

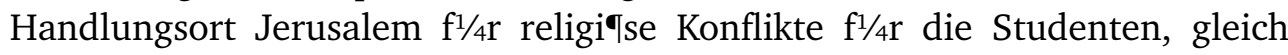
welcher Religionszugeh $\boldsymbol{T r i g k e i t}$ einen Rahmen des Bekannten und Vertrauten darstellte und ihnen somit einen Zugang zum Textinhalt erm Iglichte.

\section{Die Projektarbeit}

\subsection{Hinf $1 \frac{1}{4}$ hrung zum literarischen Text}

Das Thema $\mathrm{f}^{1 / 4} / \mathrm{r}$ die Kursarbeit im Fr¹/4hjahrssemester 2010 war "Gleichheit Andersartigkeit". Da in dieser Fortgeschrittenenklasse die Studenten 
schon mit dramapơdagogischen Elementen im Unterricht vertraut waren (Aufw $\mathrm{rm}^{1}$ /4bungen, Rollenspiele, bungen zur szenischen Improvisation), konnte gezielt mit konkreten Aktivitơten begonnen werden, die eine aktive Auseinandersetzung mit der vereinfachten Textfassung des Dramas zum Ziel hatten. Die Voraussetzungen waren geschaffen, um die Rollen zu interpretieren und mit Ausdrucks $1 / 4$ bungen zu beginnen. Die Thematik des Dramas forderte jedoch von den Studenten im Unterrichtsgeschehen ein hohes Ma an Offenheit und Akzeptanz gegen $1 / 4$ ber dem Fremden und Unbekannten. Zweifelsohne $w^{1 / 1} / 4$ rde es bei der Rollenarbeit zu Situationen kommen, in denen ein $j^{1 / 4} / 4$ discher Student die Rolle des Muslim, der Muslim die Rolle eines Christen oder der Christ die Rolle eines Juden spielen $\mathrm{m}^{1 / 4} \mathrm{sste}$. Um eine Begegnung mit dieser imaginoren, anderen Person zu initiieren, wurden gezielt bungen aus der Theaterarbeit eingesetzt:

Im Theater bietet sich die einmalige Gelegenheit unter der Maske einer als Nicht-Ich definierten Person probeweise einen virtuellen Aspekt des Selbst zu verk Trpern und sich mit ihm auseinanderzusetzen, als wore es eine Andere. (Huber 2004: 60)

Im Folgenden sollen einige Aktivitơten kurz beschrieben werden, die bei diesem Projekt als Aufworm $1 / 4$ bungen oder bei der szenischen Interpretation eingesetzt wurden und sich $\mathrm{f}^{1 / 4} / \mathrm{r}$ den Lernprozess als geeignet erwiesen. Sie f $\Psi$ rderten die Bereitschaft zur Kommunikation innerhalb der Gruppe und erleichterten das Einf $1 / 4$ hlungsverm $I g e n$ in die Figuren. Auerdem wurden die Lernenden zu spontanen sprachlichen Reaktionen herausgefordert, begleitet von K $\boldsymbol{\top}_{\mathrm{rperbewegung}}$ Mimik und Gestik. Durch die Einbeziehung dramapodagogischer Aktivitơten wird die Angst vor freiem Sprechen stark reduziert, was z.B. Huber deutlicher heraus gearbeitet hat:

Die magische Zauberformel von Stanislawsky 'Als-ob' kann man im Fall des Fremdsprachlers als Schauspieler in folgenden Satz fassen: Ich handle, als ob ich ein Schauspieler wore, ich geborde mich, als ob ich diese Person wore. Ich rede, als ob ich Deutsch k Innte. Dieser Impuls verwandelt die Angst in eine Produktionskraft, er trogt den Schauspieler 1/4ber Hemmungen hinweg und losst ihn 1/4ber die schulspezifische Behinderung des Lernens hinauswachsen. Das magische Als-ob befreit auch vor Schamgef $1 / 4$ hlen: In der Regel scheuen erwachsene Fremdsprachenlernende davor zur $1 / 4$ ck, die fremde Sprache ungeniert zu imitieren. Dank dem magischen Als-ob wird das Theater der Fremdsprache jenseits der Schamgrenze gespielt. (Huber 2004: 68)

\subsection{Gemeinsamkeiten / Unterschiede im Stuhlkreis}

Bei dieser bung werden die Studenten $\mathrm{f}^{1} / 4 \mathrm{r}$ Gemeinsamkeiten und Unterschiede sensibilisiert. Die Anweisungen, die gegeben werden, beziehen sich zunochst auf roumliche Gegebenheiten, werden dann aber auf pers $\lceil$ nliche Merkmale und Unterschiede ausgeweitet. bungsverlauf: Alle sitzen im Stuhlkreis. Es gibt einen Stuhl weniger als die Anzahl der Personen. Eine Person steht in der 
Mitte und gibt den anderen Anweisungen, den Stuhl zu tauschen (nach rechts $\mathrm{r}^{1} / 4$ cken, nach links $\mathrm{r}^{1} / 4 \mathrm{cken}$ ), er/sie muss schnell einen freien Platz fangen. Dann werden statt Richtungsanweisungen Gemeinsamkeiten definiert und nur diese Personen $\mathrm{m}^{1 / 4}$ ssen aufstehen und den Platz tauschen: alle mit Brille / alle mit Muttersprache Russisch / alle mit Jeans / mit Turnschuhen / alle, die im April Geburtstag haben ......

Die bung ist als Aufworm¹/4bung sehr geeignet, bereitet aber auch inhaltlich auf das Thema "Gemeinsamkeiten"vor.

\subsection{Blinden $1 / 4$ bung}

Die Fobigheit aufeinander $\mathrm{zu}$ h $\boldsymbol{\top}$ ren und entsprechend $\mathrm{zu}$ reagieren bildet eine Grundlage $\mathrm{f}^{1} / 4 \mathrm{r}_{\text {Einf }} 1 / 4$ hlungsverm $T$ gen und ist wesentlich $\mathrm{f}^{1} / 4 \mathrm{r}$ eine daraus resultierende Zusammenarbeit. Blinden $1 / 4$ bungen sensibilisieren auch $\mathrm{f}^{1} / 4 \mathrm{r}$ die Verantwortung, die man f1/4r den Partner trogt und passen deshalb thematisch sehr gut in einen Rahmen, in dem Teamarbeit gefordert wird. Besonders in multi-kulturellen und multi-ethnischen Gruppen bieten sich diese bungen an, denn meistens tritt spontan der berraschungseffekt ein, dass Kommunikation mit dem unbekannten Partner $\mathrm{m}$ ๆglich ist und sogar Spa macht. Ein Beispiel $\mathrm{f}^{1} / 4 \mathrm{r}$ eine Blinden $1 / 4$ bung: Zwei Partner arbeiten zusammen. A ist der Blinde, $\mathrm{B}$ ist der Blindenf $1 / 4$ hrer. B gibt A einen Laut vor (Katze, Hund, Vogel, Pfeifton, Schnalzen....), den sich der Blinde einprogen muss. Jetzt schlieen alle Blinden die Augen, die Blindenf $1 / 4$ hrer stoen ihre Kennlaute aus und entfernen sich langsam. Sie wechseln oft den Standort, entfernen sich immer weiter und stoen dabei leise ihren Ruf aus. Jeder Blinde sucht jetzt seinen Blindenf $1 / 4$ hrer, wobei seine einzige Orientierungshilfe dessen Ruf ist. Jeder F1/4hrer ist $\mathrm{f}^{1} / 4 \mathrm{r}$ die Sicherheit seines Blinden verantwortlich. Der Blinde geht nur weiter, wenn er den Ruf h⿻rt. Wenn er ihn nicht h⿻rt, bleibt er sofort stehen.

\subsection{Der F1/4nferkreis Vom Wort zur Bewegung}

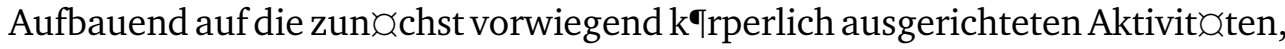
werden zunehmend sprachliche Elemente in die Lernschritte integriert. Meistens ist den Studenten die wichtige Bedeutung von Intonation, Mimik, Gestik und Bewegung im Raum in Verbindung mit sprachlichem Ausdruck nur unzureichend bewusst. Um sie daf $1 / 4 \mathrm{r}$ zu sensibilisieren eignet sich die folgende bung, in der jeweils $\mathrm{f}^{1} / 4 \mathrm{nf}$ Personen als Kleingruppe im Kreis stehen und zusammen arbeiten. A gibt ein Wort oder einen kurzen Satz aus der vereinfachten Textfassung vor. Diese verbale Aussage soll ganz neutral, wie von einem Roboter gesprochen werden. Der Nochste im Kreis (Person B) 1/4bernimmt genau diese verbale Aussage, gibt ihr aber eine bestimmte Intonation. Person $C$ holt sich genau an die beiden Vorgaben (Wort + Intonation), wiederholt den Satz, f1/4gt aber Mimik hinzu. D 1/4bernimmt und ergonzt mit einer Geste. Schlielich vervollst $\not n d i g t$ Person E das Ganze mit Bewegung im Raum.

In einer darauf aufbauenden bung werden Sprechkarten mit einem Satz 
ausgeteilt. Die Vorgaben sind jetzt nicht von den Lernenden selbst gewohlt, sondern von der Lehrperson gesteuert. Sie sind durch ihre Auswahl darauf ausgerichtet, bestimmte Gef $1 / 4$ hlsmomente des interpretierten Textes anzusprechen und zum Leben zu erwecken. Zunochst werden an der Tafel Adjektive

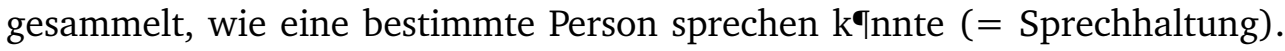
Ein Stuhl steht in der Raummitte. Ein Spieler setzt sich auf den Stuhl (Person A) und liest gonzlich neutral und ohne jegliche Intonation seinen Satz vor. Die anderen sitzen im Kreis um ihn herum. Einer nach dem anderen geht in die Kreismitte, stellt sich hinter den Sitzenden, wiederholt den Satz, gibt ihm aber eine pers nliche Intonation. Der auf dem Stuhl Sitzende schliet die Augen und wohlt dann die Darbietung, die seiner Meinung nach am besten seiner Vorgabe und damit der darzustellenden Figur aus dem Drama entspricht. Chorisches Sprechen kann sich daran anschlieen, indem Person A alle Mitspieler anleitet, den Satz nachzusprechen.

\subsection{Den Knoten II sen}

Diese bung reflektiert in gewisser Weise das zun ochst unentwirrbar scheinende Familienchaos, dem wir in dem Drama begegnen, bezieht sich aber auch inhaltlich auf eine bestimmte Textpassage: "Wie sich der Knoten, der so oft mir bange machte, nun von sich selber $1 \boldsymbol{\top}_{\text {set }}$ Gott! Wie leicht mir wird, dass ich nun weiter auf der Welt nichts zu verbergen habe!"(Nathan der Weise, 5. Aufzug, 5. Auftritt). Alle Mitspieler stehen eng aneinander im Kreis und strecken beide Arme nach vorne in die Kreismitte. Die Augen werden geschlossen. Auf ein Zeichen hin greifen alle Spieler zwei Hơnde und lassen diese nun nicht mehr los, bis der Knoten sich gel $\boldsymbol{T s}_{\mathrm{st}}$ hat. Man kann unter den Armen der anderen durchkriechen, dar $1 / 4$ bersteigen, sich drehen, alles ist erlaubt um das Ziel zu erreichen, dass der menschliche Knoten, das Wirrwarr, gel $\boldsymbol{\top}_{\mathrm{st}}$ wird. Nach gelungener Arbeit stehen alle Mitspieler in einem oder mehreren Kreisen (das Resultat ist nicht vorhersehbar) und halten sich noch immer an den Hơnden. Ein groes Erfolgserlebnis, denn allen erscheint die Situation nat $1 / 4$ rlich unl $\boldsymbol{\top}_{\text {sbar. }}$

\subsection{Standbild und Sprache}

Ein Spieler geht in die Raummitte und nimmt eine charakteristische Haltung ein, die zu einer bestimmten Person aus dem Drama passt. Mit der Haltung soll zum Ausdruck gebracht werden, wie sich die Figur gerade f1/4hlt und in welcher Situation sie sich befindet. Die Beobachter 1/4berlegen, was die Person gerade denkt. Nacheinander treten die Mitspieler hinter die Person, legen ihre Hand auf die Schulter und sprechen in 'Ich-Form' einen Satz, von dem sie glauben, dass er zu der dargestellten Situation passt. Alle hinzugetretenen Darsteller bleiben bei der Figur stehen. Der Spielleiter zeigt jetzt noch einmal auf die einzelnen Personen (wie ein Chorleiter) und diese wiederholen auf sein Zeichen hin ihren Satz.

Der Protagonist lơsst die Sơtze auf sich wirken und entscheidet, welche 
Stimmen wichtig, welche passen oder nicht passen. Der Darsteller arrangiert den Chor nach seinen Vorstellungen um. Stimmen, die nicht zu ihm passen, werden entfernt. Daran anschlieend kann das Publikum den Spielern auch Fragen stellen oder deren Haltung / Aussage kommentieren.

\subsection{Arbeit mit Rollenkarten}

Um die verworrenen Familienbeziehungen des Dramas vorzuentlasten, dient ein Familienspiel mit Rollenkarten. Alle Studenten bekommen eine Sprechkarte mit einem kurzen Text 1/4ber die darzustellende Person und stellen diese vor. Gleichzeitig gibt es zu jeder Person eine passende Sprechkarte, die die verborgene Hintergrundinformation (= das Geheimnis) dieser Person verrot. Die Studenten machen sich mit ihren Rollen vertraut und stellen sich der Gesamtgruppe vor. Dann tritt jeweils die Person mit dem entsprechenden Geheimnis hinter den Spieler und verrot 'sein Geheimnis'. Somit ist von Anfang an schon klar, dass z.B. Recha nicht die leibliche Tochter von Nathan und auch keine $\mathrm{J} 1 / 4$ din ist. Die bung erwies sich als auerordentlich effektiv, denn sie erbrachte den Lernern eine wesentliche Vorentlastung, die sie bei der weiteren Textarbeit unterst $1 / 4$ tzte und nicht entmutigte. Ich hatte mit anderen literarischen Texten die Erfahrung gemacht, dass die Studenten relativ schnell demotiviert werden, wenn beziehungsreiche inhaltliche Zusammenh onge nicht von Anfang an transparent sind.

\subsection{Augentheater}

Aufbauend auf die Arbeit an einzelnen Rollen $\mathrm{m}^{1 / 4} \mathrm{ssen}$ zunehmend bungen eingef $1 / 4$ gt werden, die das Verstơndnis von komplexeren Zusammenhơngen eines Handlungsverlaufs aus der Perspektive verschiedener Figuren f rdern. Kleingruppen bekommen den Auftrag, in 3-5 Standbildern den Inhalt einer Textpassage oder einer Szenenabfolge aus dem Drama darzustellen. Die Zuschauer schlieen die Augen, wohrend das Standbild aufgebaut wird. Auf ein akustisches Zeichen hin Iffnen sie die Augen und betrachten die ihnen dargebotene Szene. Auf ein erneutes akustisches Zeichen hin werden die Augen wieder geschlossen, wohrend die Gruppe das nochste Bild aufbaut. Das ffnen und Schlieen der Augen ist vergleichbar mit dem ffnen und Schlieen des Vorhangs auf einer B $1 / 4 \mathrm{hne}$. Die bung beinhaltet ein beachtliches Spannungspotential. Das Schlieen der Augen kreiert bei den Zuschauern eine hohe Erwartungshaltung und erm $\boldsymbol{\top}$ glicht den Darstellern, komplexe sprachliche Inhalte reduziert auf die $k \boldsymbol{\top}$ rperliche Form darzustellen. Die bung bietet auch eine ausgezeichnete Basis zum Gesprochsanlass, visuelle Information zu verbalisieren und einen Handlungsablauf zu klơren.

\subsection{Beziehung zwischen zwei Personen definieren.}

Eine andere bung zur Auseinandersetzung mit den Figuren des Dramas in unterschiedlichen Konstellationen und Situationen ist folgende Aktivit@rt: Jeder 
Spielende identifiziert sich mit einer Rolle ( $=$ Person A) und schreibt einen Satz oder eine kurze Aussage, die typisch $\mathrm{f}^{1} / 4 \mathrm{r}$ diese Person in dieser Rolle und in der gewohlten imaginoren Situation ist. Je klarer A f $1 / 4 \mathrm{r}$ sich seine Rolle definiert (Wer bin ich? Wo bin ich? Was will ich von der anderen Person?), desto leichter kann der Partner in das Rollenspiel einsteigen und das Angebot annehmen. Person A wohlt eine Person B und fordert diese zum Mitspielen auf. Beide stehen in der Raummitte $\mathrm{R}^{1 / 4} 4$ cken an $\mathrm{R}^{1 / 4}$ cken. B hat keine Ahnung, welche Rolle die hinter ihm stehende Person gleich spielt und welche Aussage ihm prosentiert wird. Sobald A bereit ist, dreht er/sie sich um, tritt vor Person B und spricht seinen/ihren Text. Dieser Text gibt Person B eine spezifische Vorgabe, auf die er/sie sprachlich spontan reagieren muss.

\subsection{Die Arbeit mit den Szenen}

Der Inhalt des Dramas wurde basierend auf der vereinfachten Textfassung in Kleingruppen in Dialogform szenisch interpretiert. Wohrend ihrer ersten Prosentationen stellten die Lernenden allerdings schnell fest, dass der verbale Ausdruck beim Spiel dominierte, weil sich fast alle an ihren geschriebenen Dialogen festhielten und der $\mathrm{k}$-rperliche Ausdruck dadurch gehemmt und oftmals gonzlich verhindert wurde. Um die Lernenden in ihrer Aussagekraft zu unterst $\mathrm{t}^{1 / 4} \mathrm{tzen}$, wurden sie aufgefordert, ihre Sprechkarten auf ein Minimum zu reduzieren und diesen kleinen Text frei auf der B1/4hne zu sprechen. Folgende Anweisung musste dabei genau befolgt werden: Sie treten genau an Punkt X auf die B1/4hne, gehen zu Punkt Y und von dort bis zur B1/4hnenmitte. Dort bleiben Sie stehen, zohlen $\mathrm{f}^{1 / 4} / \mathrm{r}$ sich langsam bis 5 , atmen ein und sprechen laut ihren Satz ins Publikum. Am Ende atmen Sie aus, zohlen wieder bis 5 und gehen in der gleichen Weise wieder von der B1/4hne. Diese Form der Bewegungsbeschreibung mag 1/4bertrieben erscheinen, bildete aber in ihrer strikten Vorgabe einen Rahmen, in dem k $\boldsymbol{\top}_{\mathrm{rperlich}}$ er Ausdruck den sprachlichen Ausdruck gezielt f $\boldsymbol{T r d e r t e}$. Die Studenten realisierten insbesondere aus ihrer Sicht als Zuschauer wohrend der Prosentation der anderen Rollen, wie wichtig die Bewegung auf der B1/4hne war und wie der dramatische Moment des sprachlichen Ausdrucks durch das zeitliche Anhalten (bis f1/4nf zohlen) erh Tht wurde. Ausgesprochen hilfreich bei dieser bung war auch die Tatsache, dass im Hintergrund leise Musik spielte. Das half sehr, die Nervositont zu verringern und den dramatischen Effekt zu steigern. Wenn das Publikum (= die Studenten) kritisierte, dass die Spieler aufder B1/4hne zu leise sprachen, wurden sie miteinem zus $₫$ tzlichen Hinweis zum nochmaligen Sprechen aufgefordert: Sprechen Sie, als wenn oben in der letzten Zuschauerreihe eine Person mit $\mathrm{H}$ Trproblemen sitzt' Oder: Schreien Sie Ihren Satz. Bei Spielern, die zu 1/4bertriebener Dramatik tendierten, wurde der Hinweis gegeben, sie sollten in der nơchsten Szene 'total langweilig' spielen. Diese Anleitung, die ich in einem Seminar von Keith Johnstone gelernt habe, wirkt bei vielen Spielern Wunder. Johnstone betont immer wieder, dass wir unsere Gef1/4hle im wirklichen Leben verstecken, wenn wir aber auf der B1/4hne stehen, glauben wir, mit Emotionen 1/4bertreiben zu 
$\mathrm{m}^{1 / 4} \mathrm{ssen}$. Die Anleitung langweilig zu spielen, bringt uns einer authentisch wirkenden Situation erstaunlicherweise nơher.

Um eine spannungslose Abfolge von Szenenbildern zu vermeiden kam die Idee auf, parallel auf zwei Darstellungsebenen zu arbeiten. Eine Ebene sollte im Hier und Jetzt sein und die Studenten in ihrer Rolle als Lernende des Deutschsprachkurses zeigen, die sich untereinander im Klassenraum 1/4ber Lessing und 1/4ber das Drama unterhalten. Dadurch sollte ein Erz,ohlrahmen dargestellt werden, der in die Szenenbilder des Dramas 1/4berleiten w $1 / 4$ rde. Diese Art der Inszenierung erschien den Studenten sehr viel interessanter als die Szenensequenzen in ihrem Ablauf dem Drama entsprechend darzustellen und gab ihnen die Mগglichkeit, die Handlungsentwicklung zu erz,ohlen, zu kommentieren oder im Gesproch durch Fragen und Antworten zu erløutern. Die Szenenbilder wurden im Theaterraum, aber auch drauen im Freien dargestellt.

Da mehrere Studenten die gleiche Rolle in unterschiedlichen Szenen spielten,

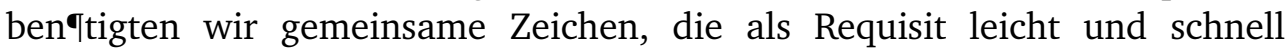
ausgetauscht werden konnten und die dargestellte Person kennzeichneten. Die Auff $1 / 4$ hrung beginnt im Theaterraum, wo alle Kleidungsst $1 / 4$ cke und Requisiten auf einer Stange aufgehơngt sind und die Spieler in Kleingruppen nacheinander, je nach Rollenzugeh Irigkeit, ihre Teile auswohlen und anziehen. Mit dieser Einf $1 / 4$ hrung der Schauspieler, die sich vor den Zuschauern mit der dargestellten Person identifizieren, werden in der Filmaufzeichnung die Spieler auch namentlich genannt.

Interessant zu beobachten war in dieser Lerngruppe die Identifikation der Studenten mit den Figuren des Dramas und ihre Wahl der Rollenverteilung. Ein christlicher Araber aus Nazareth 1/4bernahm sofort die Rolle des Patriarchen und spielte diese Rolle so $1 / 4$ berzeugend, dass niemand sonst seinen Teil 1/4bernehmen wollte und er alleine die Figur bis zum Schluss spielte. Die Darsteller des Juden Nathan und des Muslims Sultan Saladin wechselten hingegen mehrmals und es zeigten sich keinerlei Konflikte, dass ein $\mathrm{j}^{1 / 4}$ discher Student den Sultan oder ein muslimischer Student den Juden Nathan spielte. Im Gegenteil, es schien den Studenten sogar Spa zu machen, die Rolle der anderen Religion im Spiel zu 1/4bernehmen. Ich $\mathrm{f}^{1} / 4 \mathrm{hre}$ dies auf die vielfoltigen dramapodagogisch orientierten Vor $1 / 4$ bungen zur $1 / 4$ ck, in denen die Studenten zu Kommunikation und Kooperation in der Gruppe gef $1 / 4$ hrt wurden. Diese Voraussetzungen beg $1 / 4$ nstigten auch die Bereitschaft der in ihrer Anzahl im Kurs dominierenden monnlichen Kursteilnehmer in dem St¹/4ck die Rolle einer Frau zu spielen.

\subsection{Die Magie des 'dritten Auges'}

Die fortschreitende Entwicklung der Projektarbeit sollte zunouchst lediglich durch kurze Videoaufzeichnungen dokumentiert werden. Daraus entwickelte sich jedoch eine Filmaufnahme der gesamten Projektarbeit und der abschlieenden Auff $1 / 4$ hrung. Meine anfơnglichen Bedenken, dass eine laufende Filmkamera die Studenten bei ihrer Arbeit hemmen und st Iren w$^{1 / 4} / 4$ rde, wurde durch die Akzeptanz des Mediums seitens der Lernenden aufgehoben. Zu 
meiner berraschung begegnete ich der magischen Kraft 'des dritten Auges'. Die Studenten engagierten sich sehr viel mehr bei den Aufnahmen vor der laufenden Kamera als in der normalen Klassenraumsituation. Sie wurden zum lauten und deutlichen Sprechen motiviert und arbeiteten intensiver mit KIrperausdruck. Ebenso wertvoll erwiesen sich die Filmaufzeichnungen bei der Eigenevaluation. Oftmals sahen wir zu Beginn des Unterrichts Ausschnitte unserer Arbeit der vergangenen Woche und konnten deutlich durch die somit entstandene roumliche und zeitliche Distanz Schwachstellen in der eigenen Arbeit erkennen. Die Studenten brachten weiterhin zum Ausdruck, dass durch die Filmaufnahmen ihre Arbeit an Wert gewinnen $w^{1} / 4$ rde, denn die Aufzeichnungen blieben ihnen erhalten und woren auch anderen Interessierten zug onglich. Eine Auff $1 / 4 \mathrm{hrung}$ am Ende des Semesters wore somit nicht mehr das einzige Endprodukt ihrer Arbeit. Auerdem erm $\boldsymbol{\top}$ glichte das Medium Film, unterschiedliche Orte auf dem Campus in die Szenen mit einzubeziehen. Einschronkend muss ich jedoch anmerken, dass durch die Filmaufnahmen eine zus otzliche enorme Arbeitsbelastung entstanden ist. Die Filmszenen mussten technisch bearbeitet, geschnitten, mit Untertiteln, kurzen einf $1 / 4$ hrenden Texten und mit Musik unterlegt werden. Bei einem solchen Projekt sollte zu Beginn klar sein, wer von den Studenten mit dieser Art von Arbeit vertraut ist und 1/4ber die technischen Voraussetzungen verf $1 / 4$ gt. Das Endprodukt unserer Arbeit wurde schlielich eine $45 \mathrm{~min}^{1 / 4 t i g e}$ Filmproduktion. ${ }^{1}$

\section{R¹/4ckmeldung der Studenten und abschlieende Zusammenfassung}

Die Projektarbeit zielte darauf ab, durch Kooperation in der Gruppe und durch den Einsatz von K $\boldsymbol{\top}$ rpersprache, Mimik, Gestik eine intensive Auseinandersetzung mit den Figuren eines klassischen deutschen Dramas zu initiieren. Kreative Textarbeit und schriftlicher Ausdruck sollten in szenische Interpretation umgesetzt werden. Dass die Koordination und Durchf $1 / 4$ hrung der Inszenierung mit abschlieender Prosentation im Film dokumentiert wurde, war urspr $1 / 4$ nglich nicht beabsichtigt, ergab sich aber im Verlauf der Arbeit und bildete einen zus $₫$ tzlichen Lernimpuls, der allerdings dar1/4ber hinaus auch die Entwicklung technischer Forhigkeiten beinhaltete. Die R¹/4ckmeldung der Studenten entnahm ich der Umfrage, die am Technion jedes Semester vom 'Zentrum zur F 'rderung der Lehrtotigkeit' zu den belegten Veranstaltungen durchgef $1 / 4 \mathrm{hrt}$ wird. Darin bewerten die Studenten den Inhalt und die Relevanz des Kurses, den didaktischen Aufbau, die Lehrmethode und die Lehrperson. Zudem werden die Studenten auch zu pers Inlichen Aussagen aufgefordert. Diese R $1 / 4$ ckmeldung zeigt generell eine 1/4berdurchschnittlich hohe Akzeptanz der Unterrichtsform unter Einbezug von Dramatechniken. Der offene Raum, der eine aktive Form des Unterrichts und der Sozialformen erm Iglicht, wird als positiv empfunden und die lockere Lernatmosphore in Bezug gebracht zu gesteigertem Lernerfolg.

\footnotetext{
${ }^{1}$ Siehe http://humanities.technion.ac.il/Department\%20News.htm.
} 
Oft wird auch betont, wie der Zugang zur Fremdsprache durch die Einbindung von nonverbalen Anteilen der Kommunikation erleichtert wird und wie auf diesem Weg ohne die sonst trockene und erschwerende Regelvermittlung die Sprache gelernt werden kann. Die Mehrzahl der Studenten betont die Wichtigkeit von spielerischen Aufworm¹/4bungen, die sie auf das Unterrichtsgeschehen vorbereiten. Die Ergebnisse dieser Umfrage zeigen deutlich, dass dramapodagogische Elemente im Fremdsprachenunterricht sehr erfolgreich eingesetzt werden $\mathbf{k} \boldsymbol{\top} \mathrm{nnen}$, dass sie motivierend auf die Lerner wirken und dass ein $\mathrm{h}$ Therer Lernerfolg zu verzeichnen ist.

Diese generelle R $1 / 4$ ckmeldung der DaF-Studierenden im Technion fand auch in diesem spezifischen Kurs Bestortigung. Die Unterrichtsmethode hat sie sehr motiviert und ihnen vor allen Dingen Spa gemacht. Weiterhin wurde allgemein festgestellt, dass die Gruppenatmosph ore auerordentlich gut war. Die Studenten bestơtigten, dass die dramapơdagogischen Elemente beim Textverstơndnis und der Erstellung der eigenen Dialoge sehr hilfreich waren. Deutlich wird hier aber auch, dass die R1/4ckmeldungen der Studenten ausschlielich die angewandte Methodik kommentieren. Konflikte und Reibungspunkte, wie Lessing sie in seinem Drama auch $\mathrm{f}^{1} / 4 \mathrm{r}$ die heutige Zeit sehr aktuell anbietet, wurden leider nicht aufgegriffen und als Gesprochsanlass von den Studenten nicht angenommen. Nat ${ }^{1 / 4} / 4$ lich waren sich die Studenten der Thematik des Dramas und ihrer Bedeutung $\mathrm{f}^{1 / 4} / \mathrm{r}$ die heutige Zeit vollkommen bewusst, aber auch in diesem Lernkontext zeigt sich leider die allgemein bestehende Verhaltensweise des Ignorierens und des Wegschauens. Man ist sich einer bestehenden Problematik bewusst, zieht es aber vor, nicht dar $1 / 4$ ber zu reden oder gar Schritte zu unternehmen, die zu einer Veronderung f $1 / 4$ hren $k \boldsymbol{N}$ nnten. Wenn punktuell 1/4berhaupt eine Auseinandersetzung mit dem Textinhalt erfolgte, so fand diese auf eher humorvoller Ebene statt. Stand zum Beispiel in einer Szene das Thema Geld $\mathrm{f}^{1} / 4 \mathrm{r}$ den Juden Nathan im Mittelpunkt, so kam gewiss von irgendeinem $\mathrm{j}^{1 / 4} / 4$ dischen Studenten der Kommentar, 'dass Juden ja schlielich nur an Geld denken w1/4rden'. Ebenso konnte man damit rechnen, wơhrend der Berichterstattung des Sultans 1/4ber den Kampf mit den Kreuzfahrern, eine uerung von muslimischen Studenten zu h⿻ren, dass

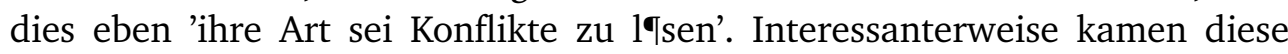
stereotypen uerungen der Selbstkritik aber immer nur aus dem eigenen Umfeld, d.h. es kam nie zu Spannungen innerhalb der Gruppe. Reibungspunkte und Konfliktsituationen wurden $\mathrm{m}$ Iglicherweise auch deshalb ausgeschaltet, weil das Theaterspiel auch einen sicheren Rahmen bieten kann, pers Inliche Konflikte zu verdecken. Das mangelnde Interesse einer inhaltlichen Auseinadersetzung mit dem Drama 1/4berraschte mich jedoch nicht, da ich schon zuvor beim Einsatz von literarischen Texten im Unterricht die Erfahrung gemacht hatte, dass Studenten einer Technischen Universitot in Israel offensichtlich nur sehr schwer Zugang zur Textinterpretation finden. $\mathrm{F}^{1 / 4} \mathrm{r}$ sie lag bei dem Projekt eindeutig der Arbeitsschwerpunkt auf sprachlichem Ausdruck, der Erweiterung ihrer Kommunikationsfohigkeit und einer guten Gruppenatmosphare beim Lernen. Dass dar $1 / 4$ ber hinaus Lessing ihnen eigentlich auch etwas anderes 
vermitteln k $\boldsymbol{I n n t e}$, blieb unbeachtet. Die Tatsache, dass sich Ali, ein Muslim aus Galiløa, im Unterricht eine Kipa, die $\mathrm{j}^{1} / 4$ dische Kopfbedeckung, aufsetzte und die Rolle von Nathan spielte und Daniel, ein Jude aus Tel Aviv die Rolle von Sultan Saladin 1/4bernahm mag schon ein Erfolg der Theaterarbeit sein, dass sich mit Hilfe der Dramapơdagogik hier eine Annơherung zur Begegnung abzeichnet. Israelis $\mathrm{m}$ Igen zwar als sehr offen, lebendig und spontan gelten, meine Erfahrung hat mir aber immer wieder gezeigt, dass bei der Behandlung spezieller Themen im Unterricht (der Konflikt zwischen Juden und Muslimen ist zweifelsohne einer davon) ein hohes $\mathrm{Ma}$ an Einf $1 / 4$ hlungsverm Igen und Diplomatie erforderlich ist, um sinnlosen Wortgefechten vorzubeugen. Deshalb $\mathrm{d} 1 / 4 \mathrm{rfen}$ meines Erachtens solche Auseinandersetzungen nicht forciert werden. Alleine die Tatsache, dass die Studenten in der Fremdsprache und ohne $\mathrm{R}^{1} / 4$ cksicht auf ethnische, religi $\Phi_{s e}$, soziale oder linguistische Voraussetzungen miteinander kommunizierten und Bereitschaft zeigten mit dem Drama zu arbeiten, ist in diesem spezifischen Kontext als Lernerfolg zu verzeichnen, auch wenn die Projektarbeit $\mathrm{m}$ ๆglicherweise nicht den klassischen Anforderungen der literarischen Textarbeit im Fremdsprachenunterricht gerecht wird.

Kritisch angemerkt wurde von den Studenten die relativ zeitintensive Auseinandersetzung 1/4ber mehrere Wochen mit einem literarischen Text. Die Studenten brachten zum Ausdruck, dass in den anderen DaF-Kursen die Lerninhalte durch mehr Abwechslung an Themen und Textsorten dargeboten wurden. Vor dem Hintergrund unserer extrem schnelllebigen Zeit, in der wir alle einer permanenten Flut insbesondere von visuellen Medien ausgesetzt sind, erscheint mir diese Kritik der Studenten verstondlich. Vereinzelt kam auch die R $1 / 4$ ckmeldung, dass Studenten sich mit der szenischen Darstellung 1/4berfordert $\mathrm{f}^{1} / 4 \mathrm{hlten}$, da sie der Meinung waren, kein schauspielerisches Talent $\mathrm{zu}$ haben. Dem ist entgegenzuhalten, dass in jedem Unterricht und bei Anwendung aller Lern- und Lehrmethoden kaum die Gesamtheit der Lerngruppe angesprochen werden kann. Immer wird es vereinzelt Lernende geben, die am Lernprozess nicht teilnehmen. Aus meiner pers $\Phi$ nlichen Erfahrung kann ich jedoch berichten, dass herk $\boldsymbol{I m m}_{\mathrm{m}}$ licher Frontalunterricht, der auf die Vermittlung von Regelwissen ausgerichtet ist, zu einem sehr viel h Theren Prozentsatz von gelangweilten, passiven und nicht motivierten Lernern $\mathrm{f}^{1} / 4 \mathrm{hrt}$. Meine Beobachtungen des fortlaufenden Unterrichtsgeschehens k Innen aber auch die wichtige Bedeutung der zeitlich intensiven Projektarbeit bestotigen. Nachdem die Studenten mit der individuellen Textarbeit und ihrer szenischen Interpretation vorangeschritten waren und die ersten Anzeichen einer gemeinsamen Darstellung des St $1 / 4$ ckes erkannten, schwand ihre gelegentliche Unlust und sie ergaben sich sozusagen 'ihrem Schicksal'. Je mehr sie sich in die Rollen vertieften und als solche miteinander in Aktion traten, desto mehr wurden sie $\mathrm{f}^{1} / 4 \mathrm{r}$ die weitere Zusammenarbeit motiviert. Ihre Begeisterung und die eigene Wertschðtzung der Arbeit gingen zum Schluss so weit, dass sie beschlossen, zur abschlieenden Prossentation auch andere Studierende einzuladen und die Auff $1 / 4$ hrung zu filmen.

Es wore zu 1/4berlegen, ob zuk¹/4nftige Projekte dieser Art mit dem gleichen Zeitaufwand innerhalb eines Semesters durchgef $1 / 4 \mathrm{hrt}$ werden sollten, oder es 
$\mathrm{m}$ Iglicherweise effektiver und gewiss mit weniger Aufwand verbunden wore, lediglich Ausschnitte eines Dramas oder eines longeren literarischen Textes als dramapodagogische Unterrichtseinheit zu gestalten und durchzuf $1 / 4$ hren.

\section{Bibliografie}

$=$

Huber, Ruth (2004): Pers Inlichkeit als Ressource: Rollenaushandlung und Gruppendynamik in theaterpødagogischen Prozessen. In: GFL Journal 1/2004, $52-72$

Lessing, Gotthold Ephraim (2008): Nathan der Weise. Hamburg: Hamburger Lesehefte Verlag

Schewe, Manfred (1993): Fremdsprache inszenieren. Zur Fundierung einer dramap Oodagogischen Lehr- und Lernpraxis. Oldenburg: Carl von Ossietzky Universitơt

Schwerdtfeger, Inge C. (2002): Gruppenarbeit und innere Differenzierung. Berlin/M1/4nchen/Leipzig/Wien/Z1/4rich/New York: Langenscheidt

Sting, Wolfgang (2003): Differenz zeigen. Interkulturelle Theaterarbeit als osthetisches Lernen. Ausz $1 / 4$ ge aus der Antrittsvorlesung an der Universitot Hamburgam 17.06.2003 http://www.hamburgerbildungsserver.de/faecher/dsp/didaktik/sting_2003. (20.02.2011) 


\section{Anhang 1: Kurze Zusammenfassung des Textes Nathan der Weise in Szenenbeschreibungen (vereinfachte Textversion $\mathrm{f} 1 / 4 \mathrm{r}$ die Arbeit mit Lernenden auf dem A2/B1-Niveau)}

Die Geschichte spielt zur Zeit des dritten Kreuzzuges, 1192 in Jerusalem. Der

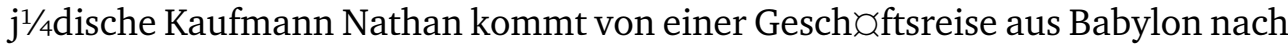
Jerusalem zur $1 / 4$ ck und erfohrt, dass sein Haus in Flammen stand und ein christlicher Tempelherr seine Tochter Recha aus dem Feuer gerettet hat.

Nathan bittet den Tempelherrn in sein Haus, damit sich seine Tochter bei ihm bedanken kann.

Der Sultan Saladin hat Geldsorgen und bittet Nathan zu sich. Er testet Nathans Weisheit und fragt ihn nach der wahren Religion. Nathan antwortet mit der Ringparabel. Saladin versteht schnell die Aussage der Gleichberechtigung der drei Religionen. Er bittet darum, Nathans Freund bleiben zu d $1 / 4$ rfen. Nathan bietet ihm an, dass er ihm Geld leihen kann, was den Sultan sehr erfreut.

Der Tempelherr hat sich in Recha verliebt und will sie heiraten, aber Nathan z'gert mit seiner Einwilligung. Daja, die Haush đlterin von Nathan, erz,ohlt dem Tempelherrn, dass Recha nicht die leibliche Tochter von Nathan ist. Er hat sie adoptiert und ihre Eltern waren Christen.

Der Templer geht zum Patriarchen von Jerusalem und erz,ohlt die Geschichte, nennt aber keine Namen; doch das Kirchenoberhaupt m $\Psi$ chte sofort den Namen des Juden wissen, damit er auf den Scheiterhaufen kommt. Durch ein Verzeichnis von einem Klosterbruder findet man heraus, dass die von Nathan erzogene Recha und der christliche Templer Geschwister sind. Der Klosterbruder hatte bei dem verstorbenen Vater von Recha ein kleines Buch gefunden, in dem alle Namen seiner nahen Verwandtschaft geschrieben waren. So findet man auch heraus, dass Assad, der verschollene Bruder von Saladin, zum Christentum konvertiert war und mit einer deutschen Frau zwei Kinder hatte: Blanda von Filnek (Recha) und Leu von Filnek (der Templer). Somit sind der Templer und Recha Geschwister und auch Nichte und Neffe des Muslims Saladin.

Saladin erkennt Assads Handschrift in dem Gebetsbuch, das der Klosterbruder bei dem gefallenen Assad gefunden hat.

Der Tempelherr ist g1 1/4cklich 1/4ber die neu gewonnene Familie und das St¹/4ck endet in wechselseitigen Umarmungen:

Ihr nehmt und gebt mir, Nathan!

Mit vollen Honden beides! Nein, Ihr gebt

Mir mehr, als Ihr mir nehmt! Unendlich mehr! (2. Aufzug, 8. Auftritt)

\section{Anhang 2: Szenentext als Grundlage zur Dialoginterpretation}

Wir sind in Jerusalem irgendwann zwischen den Jahren 1189-1192. Es ist die Zeit des dritten Kreuzzugs. Der Jude Nathan kommt von einer Geschðftsreise aus Babylon nach Jerusalem zur $1 / 4$ ck. Dort lebt er mit seiner Tochter Recha und 
seiner Haush œlterin Daja, die eine Christin ist. Daja erzøhlt ihm, dass ein Feuer im Haus war und dass Recha fast umgekommen sei. Ein christlicher Tempelherr habe sie gerettet. Recha denkt, dass ein Engel sie gerettet habe, denn der Tempelherr habe ein weies Gewand getragen.

NATHAN: Recha! Meine liebste Tochter, ich bin zur¹/4ckgekommen! Diese Geschoftsreisen machen mich ganz kaputt. Wo bist du Recha? Komm, sag deinem Vater 'Guten Tag'.

DAJA: Oh mein Gott! Das Haus ist so schmutzig!! Ich muss den ganzen Tag putzen. Wo ist blo dieses Kind?

NATHAN: Was ist passiert Daja? Warum ist alles so schmutzig?

DAJA: Oh Herr Nathan! Ein groes Ungl1/4ck ist passiert!! Ein Feuer! Ein Feuer! (Recha kommt)

RECHA: Oh Vater! Ich bin so gl1/4cklich, das ist der beste Tag meines Lebens!

DAJA: Das Kind ist verr $1 / 4$ ckt!

NATHAN: Recha, warum bist du so gl1/4cklich? Das ganze Haus ist zerst $\boldsymbol{\top}_{\mathrm{rt}}$ !

RECHA: Das Feuer, das Haus! Mir ist alles vףllig egal, ich bin verliebt!!

DAJA: Was weit du schon von Liebe?

RECHA: Ja verliebt, Vater! In einen Engel!!

DAJA: Der Tempelherr? Du nennst diesen Tempelherrn einen Engel?

\section{Anhang 3: Beispiel f1/4r eine Rollenkarte}

Ich bin Recha, die Tochter von Nathan. Mein Vater ist ein sehr guter Mann, aber er ist oft auf Geschoftsreisen. Dann bin ich alleine zu Hause mit Daja unserer Haushølterin.

Das Geheimnis von Recha: Sie war ein ganz kleines Kind, als man ih-

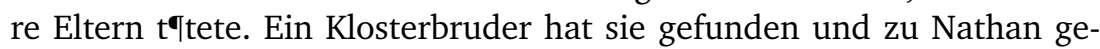
bracht. Alle wussten, dass Nathan ein sehr guter Mann war. Er war auch reich und konnte so das Kind groziehen. Nie hat er ihr aber gesagt, dass sie nicht seine leibliche Tochter ist. Sie hat auch gedacht, dass sie eine $\mathrm{J} 1 / 4 \mathrm{din}$ ist, aber in Wirklichkeit war sie eine Christin. 


\section{Anhang 4: Kommentare der Studenten}

Ïch habe meine Grundlagen in der Fremdsprache in kurzer Zeit vertieft und all dies mit einer Methode, die Spa gemacht hat."

Ïch mag diese aktive Unterrichtsmethode, sie schafft starke Interaktion zwischen den Studenten."

Prima Gruppenatmosphøre in der Klasse."

Ïch hatte nie gedacht, dass ich jemals vor einer Gruppe von Menschen etwas darstellen $\mathrm{w}^{1 / 4} / 4 \mathrm{rde}$ und dass auch noch in einer fremden Sprache! Ich stottere!"

"Diese Methoden sind wunderbar, eine Fremdsprache zu lernen."

Ëndlich kein Frontalunterricht! Ein Grund mehr, dass ich so gern zum Deutschunterricht gekommen bin. Die Methoden im Unterricht waren handlungs- und erfahrungsbezogen. Ich konnte in dieser Klasse all den anderen Lernstress vergessen."

SSo habe ich wirklich Kommunikation gelernt, nicht nur die deutsche Sprache, auch pers Inlichen Kontakt zu anderen Studenten und zur Lehrperson." 